\title{
Sur les fonctions méromorphes limites de fractions rationnelles.
}

\author{
par Nikola ObrechKofe (Sofia-Bulgaria).
}

MM. Polya, Schur, Lindwant ont démontré des théorèmes sur les fonctions entières qui sont limites de polynomes sur les zéros desquels on fait certaines suppositions. M. SAXER (1) a obtenu des résultats analogues pour des fonctions méromorphes qui sont limites de fonctions rationnelles en faisant des hypothéses sur les zéros et les põles de ces fonctions.

Récemment, M. Monter ( $\left.{ }^{2}\right)$ a démontré des théorèmes pour les fractions rationnelles à termes entrelacés sur l'axe réel et pour les fonctions méromorphes, qui sont limites de telles fractions.

Dans ce travail, dont j'ai donné $\left({ }^{3}\right)$ un résumé dans une note publiée dans les «Comptes Rendus» de l'Académie des Sciences de Paris, je considère des fonctions méromorphes qui sont limites de fractions rationnelles, sur les pôles desquelles seulement on fait des hypothèses convenables. Les résultats que j'obtiens contiennent les résultats des auteurs cités.

Nons avons le théorème suivant:

I. Supposons que la suite de fractions rationnelles

$$
R_{n}(z)=\sum_{\nu=1}^{n} \frac{A_{n \nu}}{z-\alpha_{n \nu}}
$$

tende uniformément vers une fonction méromorphe $\mathrm{R}(\mathrm{z})$, dont le point $\mathrm{z}=0$ est un point régulier. Supposons encore que l'on ait uniformément pour $\mathbf{n}$

$$
\sum_{\nu=1}^{n}\left|\frac{A_{n \nu}}{\alpha_{n+\nu}^{k+1}}\right|<M
$$

(1) W. SAXER, Ueber die Verteilung der Nullstellen und Pole von rationalen Funktionen konvergente Folgen, "Mathematische Zeitschrift *, 27, 1928, pp. 518-585.

(*) P. Montel, Sur me classe de fonctions méromorphes, "Comptes Rendus , 195, 1932, p. 643 .

(3) N. OBREchKoFF, Sur des fonctions méromorphes qui sont limites de fractions rationnelles, "Comptes Rendus», 196, 1933, p. 746. 
oit $\mathrm{k}>0, \mathrm{M}$ est un nombre fini. La fonction $\mathrm{R}(\mathrm{z})$ a la forme

$$
R(z)=P(z)+\sum_{n=1}^{\infty} A_{n}\left(\frac{1}{z-\alpha_{n}}+\frac{1}{\alpha_{n}}+\frac{z}{\alpha_{n}{ }^{2}}+\ldots+\frac{z^{n-1}}{\alpha_{n}{ }^{m}}\right),
$$

où la série $\sum_{\mathrm{n}=1}^{\infty}\left|\frac{\mathrm{A}_{\mathrm{n}}}{\alpha_{\mathrm{n}}^{\mathrm{k} \cdot 1}}\right|$ est convergente. $\mathrm{P}(\mathrm{z})$ est un polynome de degré $\mathrm{p}$ qui est égal à $[\mathrm{k}]$ si $\mathrm{k}$ n'est pas un nombre entier, et égal à $\mathrm{k}$ si ce nombre est entier; $\mathrm{m}=[\mathrm{k}]+1$ dans le premier cas el $\mathrm{m}=\mathrm{k}$ dans le second cas. Cette condition est aussi suffisante.

Nous faisons la remarque suivante: dans l'énoncé des théorèmes on pent supposer que la convergence est seulement uniforme dans le plan ouvert en enlevant les points des cercles décrits autour des poles $\alpha_{n}$ avec des rayons arbitrairement petits.

Puisque les fonotions $R_{n}($ (f) tendent vers $R(z)$, si nous decrivons autour de chaque pôle $\alpha_{q}$ de la fonction $R(z)$ une circonférence $C_{q}$ de rayon arbitrairement petit $\delta>0$, tous les pôles $\alpha_{n v}, y=\lambda_{q}+1, \lambda_{q}+2, \ldots, \lambda_{q+1} q u i$ tendent vers $\alpha_{q}$ se trouveront pour $u>N(\delta)$ dans $C_{q}$. De la formule

on conclut que

$$
\lim _{n \rightarrow \infty} \int_{C_{q}} R_{n}(z) d z=\int_{C_{q}} R(z) d z
$$

$$
\lim _{n \rightarrow \infty} \sum_{\nu=\lambda_{q}+1}^{\lambda_{q+1}} A_{n \nu}=A_{q}
$$

On voit facilement que pour chaque $g \geqq 0$ on a

$$
\lim _{n \rightarrow \infty} \underset{n=\lambda_{q}+1}{\lambda_{q+1}} \frac{A_{n v y}}{\alpha_{n y}^{g}}=\frac{A_{q}}{\alpha_{q}^{g}} .
$$

En effet, nous avons pour $\left|\alpha_{q}\right|<T,\left|\alpha_{n \mu}\right|<T$

$$
\frac{1}{T^{k+1}} \underset{v=\lambda_{q}+1}{\stackrel{\lambda_{q+1}}{\sum}}\left|A_{n v}\right|<\underset{v=\lambda_{q}+1}{\lambda_{q+1}}\left|\frac{A_{n v}}{\alpha_{n v}^{k+1}}\right|<M, \underset{v=\lambda_{q}+1}{\sum_{q+1}^{\lambda_{n}}}\left|A_{n v}\right|<M T^{k+1}=M_{1} .
$$

En écrivant la différence

sous la forme

$$
\sum_{v=\lambda_{q}+1}^{\lambda_{q}+1} \frac{A_{n q}}{\alpha_{n y}^{g}}-\frac{A_{q}}{\alpha_{q}^{g}}
$$

$$
\underset{v=\lambda_{q}+1}{\lambda_{q+1}+1} A_{n v}\left(\frac{1}{\alpha_{n y}^{g}}-\frac{1}{\alpha_{q}^{g}}\right)+\frac{1}{\alpha_{q}^{g}}\left(\sum_{\nu=\lambda_{q}+1}^{\lambda_{q+1}} A_{n y}-A_{q}\right)
$$

on démontre facilement la relation (5). 
Les pòles $\alpha_{q}$ de la fonction $R(z)$ sont simples. En effet, considérons l' integrale $(n>N=N(\delta))$

$$
j_{n}=\int_{C_{q}}\left(z-\alpha_{q}\right)^{v} R_{n}(z) d z=\sum_{\nu=\lambda_{q}+1}^{\lambda_{q+1}} A_{n \nu} \int_{C_{q}} \frac{\left(z-\alpha_{q}\right)^{\nu}}{z-\alpha_{n \nu}},
$$

$r$ étant un nombre entier $\geqq 1$. En écrivant $\left(z-\alpha_{q}\right)^{p}$ sous la forme

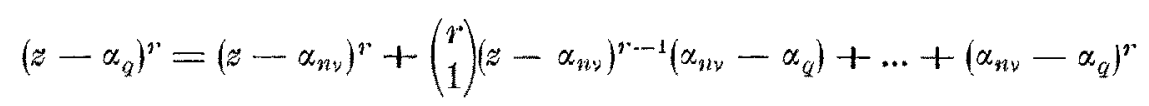

on obtient

$$
j_{n}=\sum_{\nu=\lambda_{q}+1}^{\lambda_{q+1}} A_{n \nu} \int \frac{\left(\alpha_{n \nu}-\alpha_{q}\right)^{v}}{z-\alpha_{n \nu}} d z
$$

et nous avons $\lim _{n \rightarrow \infty} j_{n}=0$. Done

$$
\lim _{n \rightarrow \infty} j_{n}=\int_{C_{q}}\left(z-\alpha_{q}\right)^{r} R(z) d z=0, \quad r \geqq 1,
$$

ce qui montre que les pòles $x_{q}$ sont simples.

Nous démontrons maintenant que la série

$$
\sum_{n=1}^{\infty}\left|\frac{A_{n}}{\alpha_{n}^{k+1}}\right|
$$

est convergente. Pour chaque nombre $m$ fixe nous avons

$$
\sum_{q=1}^{m}\left|\sum_{\nu=\lambda_{q}+1}^{\lambda_{q+1}} \frac{A_{w \nu}}{\alpha_{n \nu}^{k+1}}\right|<M,
$$

d'où en posant $n \rightarrow \infty$ et de (4) on obtient

$$
\sum_{q=1}^{m}\left|\frac{A_{q}}{\alpha_{q}^{k+1}}\right| \leqq M
$$

pour chaque $m$, ce qui démontre la convergence de la série (6).

Maintenant on voit facilement que pour chaque $g>k+1$ on a

$$
\lim _{n \rightarrow \infty} \sum_{\nu=1}^{n} \frac{A_{n v}}{\alpha_{n \nu}^{g}}=\sum_{p=1}^{\infty} \frac{A_{p}}{\alpha_{p}^{g}}
$$

Soit $\varepsilon>0$ un nombre arbitrairement petit et soit le nombre $m$ choisi de façon que pour $p>m$ on ait

$$
\left|\frac{1}{\alpha_{p}^{g-k-1}}\right|<\frac{\varepsilon}{M}, \quad\left|\frac{1}{\alpha_{n \nu}^{g-k-1}}\right|<\frac{\varepsilon}{M}, \quad \nu>\lambda_{m+1} .
$$


Nous avons

$$
\left|\sum_{p=m+1}^{\infty} \frac{A_{p}}{\alpha_{p}^{g}}\right|<\frac{\varepsilon}{M} \sum_{p=m+1}^{\infty}\left|\frac{A_{p}}{\alpha_{p}^{k+1}}\right|<\varepsilon, \quad\left|\sum_{p=\lambda_{m+1}}^{\infty} \frac{A_{n p}}{\alpha_{n p}^{g}}\right|<\frac{\varepsilon}{M} \sum_{\nu=1}^{n}\left|\frac{A_{n \nu}}{\alpha_{n \nu}^{k+1}}\right|<\varepsilon,
$$

et de (5) on conclut que

$$
\varlimsup_{n \rightarrow \infty}\left|\sum_{\nu=1}^{n} \frac{A_{n \nu}}{\alpha_{n \nu}^{g}}--\sum_{p=1}^{\infty} \frac{A_{p}}{\alpha_{p}^{g}}\right|<2 \varepsilon
$$

ce qui démontre la formule (7).

Soit $k$ un nombre non entier et écrivons $\left({ }^{4}\right)$

$$
\begin{gathered}
R_{n}(z)=-\sum_{\nu=1}^{n} \frac{A_{n \nu}}{\alpha_{n \nu}}-z \sum_{\nu=1}^{n} \frac{A_{n \nu}}{\alpha_{n \nu}^{2}}-\ldots-z^{m-1} \sum_{\nu=1}^{n} \frac{A_{n \nu}}{\alpha_{n \nu}^{m}}+ \\
\quad+\sum_{\nu=1}^{n} A_{n \nu}\left(\frac{1}{z-\alpha_{n \nu}}+\frac{1}{\alpha_{n \nu}}+\frac{z}{\alpha_{n \nu}^{2}}+\ldots+\frac{z^{n-1}}{\alpha_{n \nu}^{m}}\right) .
\end{gathered}
$$

La suite $R_{i l}(z)$ étant convergente pour $z=0$, du développement

$$
R_{n}(z)=-\sum_{p=1}^{\infty} z_{\nu=1}^{p} \sum_{\nu}^{n} \frac{A_{n \nu}}{\alpha_{n \nu}^{p+1}}
$$

il suit que $-\sum_{\nu=1}^{n} \frac{A_{n v}}{\alpha_{n \nu}^{p+1}}$ tend vers une limite $c_{p}$. Considérons alors la fonction

$$
U(z)=c_{0}+c_{1} z+\ldots+c_{m-1} z^{m-1}+\sum_{n=1}^{\infty} A_{n}\left(\frac{1}{z-\alpha_{n}}+\frac{1}{\alpha_{n}}+\frac{z}{\alpha_{n}{ }^{2}}+\ldots+\frac{z^{m-1}}{\alpha_{n}{ }^{m}}\right) .
$$

Nous démontrerons que $\lim _{n \rightarrow \infty} R_{n}(z)=U(z)$ et le théorème I sera démontré.

Pour cela nous démontrerons que la fonction

$$
V_{n}(z)=\sum_{\nu=1}^{n} A_{n \nu}\left(\frac{1}{z-\alpha_{n \nu}}+\frac{1}{\alpha_{n \nu}}+\frac{z}{\alpha_{n \nu}^{2}}+\ldots+\frac{z^{m-1}}{\alpha_{n \nu}^{m}}\right)=\sum_{\nu=1}^{n} \frac{A_{n \nu} z^{m}}{\alpha_{n \nu}^{m}\left(z-\alpha_{n \nu}\right)}
$$

tend vers la fonction

$$
V(z)=\sum_{p=1}^{\infty} A_{p}\left(\frac{1}{z-\alpha_{p}}+\frac{1}{\alpha_{p}}+\frac{z}{\alpha_{p}^{2}}+\cdots+\frac{z^{m-1}}{\alpha_{p}^{m}}\right)=\sum_{p=1}^{\infty} \frac{A_{p} z^{m}}{\alpha_{p}^{m}\left(z-\alpha_{p}\right)},
$$

dans chaque cercle $|z| \leqq R$, en enlevant naturellement les points des petits cercles décrits autour des pôles $\alpha_{p}$. En effet, soit $\varepsilon>0$ un nombre arbitraire et soit $\lambda>2$ choisi de façon que l'on ait

$$
\frac{4 M K^{m}}{\lambda^{\delta} R^{\delta}}<\varepsilon, \quad \delta=m-k>0
$$

(4) Voir aussi P. Montel, Sur les familles normales de fonctions analytiques, "Annales scientifiques de l'École Normale Supérieure », t. 33 (1916), pp. 222-302. 
Soient $\alpha_{1}, \alpha_{2}, \ldots, \alpha_{g}$ les pôles de la fonction $V(z)$ qui se trouvent dans le cercle $|z| \leqq \lambda R$ et soient $\alpha_{n_{1}}, \alpha_{n_{2}}, \ldots, \alpha_{n_{n}, n_{g}}$ les pôles de la fonction $V_{n}(z)$ qui tendent vers les premiers pour $n \rightarrow \infty$. Alors, d'après (7), on démontre facilement que

$$
\lim _{n \rightarrow \infty} \sum_{y=1}^{n_{g}} \frac{A_{n v} z^{m}}{\alpha_{n y}^{m}\left(z-\alpha_{n y}\right)}=\sum_{\nu=1}^{g} \frac{A_{y} z^{m}}{\alpha_{y}^{m}\left(z-\alpha_{\nu}\right)}
$$

Pour les põles qui sont en dehors du cercle $|z|=\lambda R$, on aura, $(n>N)$

$$
\left|z-\alpha_{n \nu}\right| \geq \frac{\lambda-1}{\lambda}\left|\alpha_{n \nu}\right|>\frac{1}{2}\left|\alpha_{n \nu}\right|, \quad\left|z-\alpha_{\nu}\right|>\frac{1}{2}\left|\alpha_{\nu}\right|
$$

done

$$
\begin{gathered}
\left|\sum_{\nu=n_{g}+1}^{n} \frac{A_{n \nu} z^{m}}{\alpha_{n v}^{m}\left(z-\alpha_{n \nu}\right)}\right|<2 R^{m} \sum_{\nu=n_{y}+1}^{n}\left|\frac{A_{n \nu}}{\alpha_{n \nu}^{m+1}}\right|<\frac{2 R^{m}}{\lambda^{\delta} R^{\delta}} \sum_{y=n_{g}+1}^{\sum_{\nu}}\left|\frac{A_{n \nu}}{\alpha_{n \nu}^{k+1}}\right|<\frac{2 R^{m} M}{\lambda^{\delta} R^{\delta}}<\frac{\varepsilon}{2}, \\
\left|\sum_{\nu=g+1}^{\infty} \frac{A, z^{m}}{\alpha_{\nu}^{m}\left(z-\alpha_{\nu}\right)}\right|<2 R^{m} \sum_{\nu=g+1}^{\infty}\left|\frac{A_{\nu}}{\alpha_{\nu}{ }^{m+1}}\right|<\frac{2 R^{m} M}{\lambda^{\delta} R^{\delta}}<\frac{\varepsilon}{2} .
\end{gathered}
$$

Les inégalités obtenues nous montre que $\overline{\lim }_{n \rightarrow \infty}\left|V_{n}(z)-V(z)\right| \leqq \varepsilon, \varepsilon$ arbitraire, c'est-à-dire que $\lim _{n \rightarrow \infty} V_{n}(z)=V(z)$.

Soit maintenant $k$ un nombre entier. Nous écrivons la fonction $R_{n}(z)$ sous la forme

$$
\begin{aligned}
R_{\nu,}(z) & =-\sum_{\nu=1}^{n} \frac{A_{n \nu}}{\alpha_{n \nu}}-z \sum_{\nu=1}^{n} \frac{A_{n \nu}}{\alpha_{n \nu}^{2}}-\ldots-z^{m} \sum_{\nu=1}^{n} \frac{A_{n \nu}}{\alpha_{n \nu}^{m-1}}+ \\
& +\sum_{\nu=1}^{n} A_{n \nu}\left(\frac{1}{z-\alpha_{n \nu}}+\frac{1}{\alpha_{n \nu}}+\ldots+\frac{z^{m}}{\alpha_{n \nu}^{m+1}}\right),
\end{aligned}
$$

et nous considerons la fonction

$$
U(z)=c_{0}+c_{1} z+\ldots+c_{m} z^{n}+\sum_{\nu=1}^{\infty} A_{\nu}\left(\frac{1}{z-\alpha_{\nu}}+\frac{1}{\alpha_{\nu}}+\frac{z}{\alpha_{\nu}{ }^{2}}+\ldots+\frac{z^{m}}{\alpha_{\nu}{ }^{m+1}}\right) .
$$

Comme ci-dessus, on démontre que $\lim _{n \rightarrow \infty} R_{n}(z)=U(z)$. Puisque la série

$$
\sum_{p=1}^{\infty} \frac{A_{p}}{\alpha_{p}^{m+1}}
$$

est convergente, cette fonction peut s'écrire

$$
U(z)=c_{0}+c_{1} z+\ldots+c_{m}^{\prime} z^{m}+\sum_{p=1}^{\infty} A_{p}\left(\frac{1}{z-\alpha_{p}}+\frac{1}{\alpha_{p}}+\frac{z}{\alpha_{p}^{2}}+\ldots+\frac{z^{m-1}}{\alpha_{p}^{m}}\right)
$$

et le théorème est démontré. 
Maintenant nous allons démontrer la partie inverse du théorème I. Soit done $R(z)$ une fonction méromorphe de la forme

(8) $R(z)=c_{0}+c_{1} z+\ldots+c_{n-1} z^{m-1}+\sum_{\nu=1}^{\infty} A_{\nu}\left(\frac{1}{z-\alpha_{\nu}}+\frac{1}{\alpha_{\nu}}+\frac{z}{\alpha_{\nu}{ }^{2}}+\ldots+\frac{z^{m-1}}{\alpha_{\nu}{ }^{m}}\right)$

où la série $\sum_{\nu=1}^{\infty}\left|\frac{A_{\nu}}{\alpha_{\nu}^{k+1}}\right|$ est convergente, $k+1 \geqq m$. Considérons la fonction rationnelle

$$
R_{n}(z)=\sum_{p=1}^{m} \frac{c_{p} z^{p-1}}{1+\frac{c_{p} z^{p}}{n}}+\sum_{\nu=1}^{n} A_{\nu}\left(\frac{1}{z-\alpha_{\nu}}+\frac{\frac{1}{\alpha_{\nu}}}{1+\frac{z}{\alpha_{\nu} N}}+\ldots+\frac{\frac{z^{m-1}}{\alpha_{\nu}{ }^{m}}}{1+\frac{z^{m}}{m \alpha_{\nu}{ }^{m} N}}\right) .
$$

On peut facilement voir qu'on peut choisir la croissance de $N$ vers l'infini avee $n$ de façon que l'on ait

$$
\lim _{n \rightarrow \infty} R,(z)=R(z)
$$

uniformément. En effet, le premier membre de la partie droite de $R_{n}($ () tend vers le membre respectif de $R($ (e). La différence entre

$$
\sum_{\nu=1}^{n} \dot{A}_{\nu}\left(\frac{1}{z-\alpha_{\nu}}+\frac{1}{\alpha_{\nu}}+\frac{z}{\alpha_{\nu}{ }^{2}}+\ldots+\frac{z^{m-1}}{\alpha_{\nu}{ }^{m}}\right)
$$

et le second membre de $R_{n}(z)$ est en valeur absolue égal an plus à

$$
\frac{1}{N} \sum_{\nu=1}^{n}\left|A_{\nu}\right| \cdot\left|\sum_{p=1}^{m} \frac{\frac{z^{2 p-1}}{p \alpha_{\nu}^{2 p}}}{1+\frac{z^{p}}{p \alpha_{\nu}{ }^{p} N}}\right|
$$

et pour chaque $R,|z| \leqq R$ on peut choisir évidemment $N$ de façon que cette expression tende vers zéro, lorsque $n, N \rightarrow \infty$. Mais on a

$$
\frac{c_{q} z^{q-1}}{1+\frac{c_{q} z^{q}}{n}}=\sum_{\nu=1}^{q} \frac{n}{z-\omega_{\nu}^{(q)} \delta_{q}}
$$

où $\omega_{\nu}^{(q)}$ sont les racines de l'équation $z^{q}=-1$ et $\delta_{q}=\left|\frac{n}{c_{q}}\right|^{\frac{1}{q}}$. Done le premier membre de la partie droite a la forme

$$
\sum_{p=1}^{n} \frac{c_{p} z^{p-1}}{1+\frac{c_{p} z^{p}}{n}}=\sum_{p=1}^{m} \sum_{p=1}^{p} \frac{n}{z-\omega_{\nu}{ }^{p p)} \delta_{p}}
$$


et la somme $\sum_{p=1}^{m} \sum_{y=1}^{p} \frac{n}{\left|\omega_{y}^{(p)} \delta_{p}\right|^{k+1}}$ est évidemment bornée. Analoguement nous avons

$$
\frac{\frac{z^{p-1}}{\alpha_{\nu}{ }^{p}}}{1+\frac{z^{p}}{p \alpha_{\nu}{ }^{p} N}}=\sum_{h=1}^{p} \frac{N}{z-\omega_{h}^{(p)} \alpha_{p}{ }^{\prime}}, \quad p \leqq m
$$

où. $\omega_{h}^{(p)}$ sont les racines de l'équation $z^{p}=-1$, et $\alpha_{p}^{\prime}=p^{\frac{1}{p}} \alpha_{y} N^{\frac{1}{p}}$. La somme correspondante pour les pôles dans le second membre de (9) est égale à la somme

$$
\sum_{\nu=1}^{n} \frac{N\left|A_{y}\right|}{p^{\frac{k+1}{p}}\left|\alpha_{\nu}\right|^{k+1} N^{\frac{k+1}{p}}}, \quad p \leqq m,
$$

et puisque $k+1 \geqq m$, il est évident qu'elle est bornée. Donc le théorème est complètement démontré.

En suivant la mème marohe de démonstration, on obtient le théorème plus général:

II. Soit

$$
R_{n}(z)=P_{n}(z)+\sum_{\mu=1}^{n} \frac{A_{n \mu}}{z-\alpha_{n \mu}}
$$

une suite de fonctions rationnelles, oì $\mathrm{P}_{\mathrm{n}}(\mathrm{z})$ sont des polynomes de degré égal au plus à $q$, et supposons qu'il existe un nombre $\mathrm{k} \geqq 0$, tel que

$$
\sum_{\mu=1}^{n}\left|\frac{A_{n \mu}}{\alpha_{n \mu}^{k+1}}\right|<M
$$

Si la suite $\mathrm{R}_{\mathrm{n}}(\mathrm{z})$ converge uniformément vers une fonction méromorphe $\mathrm{R}(\mathrm{z})$, dont le point $\mathrm{z}=0$ est régulier, elle aura la forme suivante

où la série

$$
R(z)=P(z)+\sum_{n=1}^{\infty} A_{n}\left(\frac{1}{\alpha_{n}}+\frac{1}{\alpha_{n}}+\frac{z}{\alpha_{n}^{2}}+\ldots+\frac{z^{n-1}}{\alpha_{n}{ }^{n}}\right)
$$

$$
\sum_{n=1}^{\infty}\left|\frac{A_{n}}{\alpha_{n}^{k+1}}\right|
$$

est convergente. $\mathrm{P}(\mathrm{z})$ est un polynome de degré $\mathrm{g}$ égal à max. $(q,[\mathrm{k}])$ si $\mathrm{k}$ est un nombre non entier, et $\mathrm{g}=$ max. $(\mathrm{q}, \mathrm{k})$ si $\mathrm{k}$ est entier, $\mathrm{m}=[\mathrm{k}]+1$ dans le premier cas et $\mathrm{m}=\mathrm{k}$ dans le second cas.

Nous considérons maintenant une classe spéciale de fonctions méro. morphes, limites de fractions rationnelles dont les résidus $A_{n \mu}$ sont des nombres positifs, en faisant pour les pôles des simples hypothèses. 
III. Soit

$$
R_{n}(z)=\sum_{\mu=1}^{n t} \frac{A_{n \mu}}{z-\alpha_{n i \mu}}
$$

une suite de fonctions rationnelles, $A_{n \mu}>0, \mu=1,2, \ldots, n$, les pốles $\alpha_{n \mu}$ se trouvent dans un secteur A dont le sommet est le point $\mathrm{z}=0$ et d'ouver. ture $<\pi$. Désignons par $\overline{\mathrm{A}}$ le secteur symétrique de A par rapport d̀ l'axe réel. Alors la condition nécessaire et suffisante pour que la fonction méro. morphe $\mathrm{R}(\mathrm{z})$ soit la limite uniforme de fonclions rationnelles de cette forme consiste en ce qu' elle doit avoir la forme suivante

$$
\mathrm{R}(\mathrm{z})=-\delta+\sum_{n=1}^{\infty} \frac{A_{n}}{z-\alpha_{n}}, \quad A_{n}>0
$$

où la série $\sum_{n=1}^{\infty} \frac{A_{n}}{\left|\alpha_{n}\right|}$ est convergente, $\alpha_{n}$ se trouvent dans $\mathrm{A}$ et $\delta$ est un point de $\bar{A}$.

D'après les conditions du théorème on peut choisir un nombre $\theta$ de façon que les arguments des nombres $e^{i \theta} \alpha_{n \mu}$ se tronvent entre $-\alpha$ et $\alpha$, $\alpha<\frac{\pi}{2}$, c'est-à-dire

$$
\left|e^{-i \theta} \frac{1}{\alpha_{n i \mu}}\right| \leqq \frac{1}{\cos \alpha} R\left(e^{-i \theta} \frac{1}{\alpha_{z_{i j} i}}\right)
$$

Nous avons démontré que la somme

$$
\sum_{\mu=1}^{\infty} \frac{A_{n \mu}}{\alpha_{n \mu}}
$$

tend vers une limite déterminée lorsque $n \rightarrow \infty$. Done de (11) on conclut que la somme $\sum_{\mu=1}^{n} \frac{A_{n \mu}}{\left|\alpha_{n \mu}\right|}$ est uniformément bornée. Nous avons aussi démontré que la fonction

$$
R_{i,}(z)=-\sum_{\mu=1}^{n} \frac{A_{n \mu}}{\alpha_{n \mu}}+\sum_{\mu=1}^{n} A_{n \mu}\left(\frac{1}{z \quad \alpha_{n \mu}}+\frac{1}{\alpha_{n \mu}}\right)
$$

tend vers la fonction

$$
V(z)=c_{0}+\sum_{y=1}^{\infty} A_{\nu}\left(\frac{1}{z-\alpha_{y}}+\frac{1}{\alpha_{\nu}}\right)=-\lim _{n \rightarrow \infty} \sum_{\nu=1}^{n} \frac{A_{n \mid k}}{\alpha_{n \mid \nu}}+\sum_{\nu=1}^{\infty} \frac{A_{\nu}}{\alpha_{\nu}}+\sum_{\nu=1}^{\infty} \frac{A_{\nu}}{z-\alpha_{\nu}} .
$$

Soit $\varepsilon>0$ un nombre arbitrairement petit et soit $N$ choisi de façon que l'on ait

$$
\sum_{\nu=N+1}^{\infty} \frac{A_{\nu}}{\left|\alpha_{y}\right|}<\varepsilon
$$


ce qui est possible à cause de la convergence de la série $\Sigma \frac{A_{\nu}}{\left|\alpha_{\nu}\right|}$. Nous avons vu qu'il existe des nombres $\lambda_{n}$, tels que

$$
\lim _{n \rightarrow \infty} \sum_{y=1}^{\lambda_{n}} \frac{A_{n v}}{\alpha_{n v}}=\sum_{\nu=1}^{N} \frac{A_{y}}{\alpha_{y}}
$$

Donc le nombre $\delta$ dans le théorème est egal à

$$
\delta=\lim _{n \rightarrow \infty} \sum_{\nu=\lambda_{n+1}+1}^{n} \frac{A_{n v}}{\alpha_{n v}}+\eta=g+\eta
$$

où $|\eta|<\varepsilon$. Puisque le nombre $g$ est un nombre de $\bar{A}$ et $\varepsilon$ est arbitraire. ment petit $\hat{o}=g$, le théorème est démontré.

Pour démontrer la deuxième partie du théorème, considérons les fonctions rationnelles

$$
R_{n}(z)=\frac{n}{z-\frac{n}{\delta}}+\sum_{\nu=1}^{n} \frac{A_{v}}{z-\alpha_{\nu}},
$$

qui ont la forme demandée. Nous avons $\lim _{n \rightarrow \infty} R_{n}(z)=R(z), \quad R(z)$ étant la fonction (10), et la démonstration est finie.

De la même manière on démontre le théorème suivant:

III'. Supposons que la suite des fractions

$$
R_{n}(z)=-g_{n}+\sum_{y=1}^{n} \frac{A_{n \nu}}{z-\alpha_{n y}}, \quad A_{n y}>0,
$$

où $\overrightarrow{\mathrm{g}}_{\mathrm{n}}, \alpha_{n \psi}$ sont des nombres d'un secteur A d'ouverture $<\pi$, tende vers une fonction méromorphe $\mathrm{R}(\mathrm{z})$. La fonction $\mathrm{R}(\mathrm{z})$ a la forme (10) ou $\delta$ et $\alpha_{\mathrm{n}}$ sont des nombres de $\overline{\mathbf{A}}$ et la série $\sum_{\mathbf{n}=1}^{\infty} \frac{\mathrm{A}_{\mathbf{n}}}{\left|\boldsymbol{\alpha}_{\mathbf{n}}\right|}$ est convergente, $\mathrm{A}_{\mathbf{n}}>0$.

Considérons maintenant une classe de fonctions rationnelles dont les pôles se tronvent dans un demi-plan, qu' on peut évidemment supposer le plan $j(z) \geq 0\left({ }^{5}\right)$. Nous démontrons le théorème suivant:

IV. Supposons que la suite de fonctions rationnelles

$$
R_{n}(z)=\sum_{\nu=1}^{n} \frac{A_{n v}}{z-\alpha_{n v}}
$$

où $\mathrm{A}_{\mathrm{nv}}>0, \mathrm{j}\left(\alpha_{\mathrm{n}}\right) \geqq 0$, tende vers la fonction méromorphe $\mathrm{R}(\mathrm{z})$. La fonction $\mathrm{R}(\mathrm{z})$

( $\left.{ }^{5}\right)$ Par $j(z)$ nous désignons la partie imaginaire de $z$. 
a la forme suivante:

$$
R(z)=-\gamma z+\delta+\sum_{n=1}^{\infty} A_{n}\left(\frac{1}{z-\alpha_{n}}+\frac{1}{\alpha_{n}}\right), \quad A_{n}>0,
$$

la série $\sum_{\mathrm{n}=1}^{\infty} \frac{\mathrm{A}_{\mathrm{n}}}{\left|\alpha_{\mathrm{n}}\right|^{2}}$ est convergente, $\mathrm{j}\left(\alpha_{\mathrm{n}}\right) \geqq 0, \gamma$ est un nombre réel $\geqq 0$, la série $-\sum_{\mathrm{n}=1}^{\infty} \mathrm{j}\left(\frac{\mathrm{A}_{\mathrm{n}}}{\alpha_{\mathrm{n}}}\right)$ est convergente et sa somme est égale au plus à $\mathrm{j}(\delta)$. Celte condition pour $\mathrm{R}(\mathrm{z})$ est aussi suffisante.

Soit $a>0$ un nombre arbitraire tel que $R(z)$ soit régulière pour $|z| \leqq a$, ce qui est possible parce que $z=0$ est un point régulier. La suite des fonctions rationnelles

$$
R_{n}(z-\alpha)=\sum_{\nu=1}^{n} \frac{A_{n \nu}}{z-\left(\alpha_{n \nu}+a\right)}
$$

tend uniformément vers la fonetion

c'est-à-dire

$$
R(z-a)=c_{0}^{\prime}+c_{1}^{\prime} z+c_{2}^{\prime} z^{2}+\ldots, \quad|z| \leqq p,
$$

$$
\lim _{n \rightarrow \infty} \sum_{\nu=1}^{n} \frac{A_{n \nu}}{\alpha_{n \nu}+\alpha}=-c_{\diamond}^{\prime}
$$

Parce que

$$
-j\left(\frac{A_{n \nu}}{\alpha_{n \nu}+a}\right)=A_{n \nu} \frac{j\left(\alpha_{n y}+a\right)}{\left|\alpha_{n \nu}+a\right|^{2}} \geqq a \frac{A_{n \nu}}{\left|\alpha_{n \nu}+a\right|^{2}}
$$

on a uniformément

$$
\sum_{v=1}^{n} \frac{A_{n v}}{\left|\alpha_{n v}+\alpha\right|^{2}}<M_{1}
$$

Alors comme ci-dessus on démontre que la série

$$
\sum_{y=1}^{\infty} \frac{A_{y}}{\left|\alpha_{y}+a\right|^{2}}
$$

est convergente, d'où il s'ensuit que la série

$$
\sum_{\nu=1}^{\infty} \frac{A_{\nu}}{\left|\alpha_{\nu}\right|^{2}}
$$

est convergente. Alors, en se basant sur le théorème I, on conolut que la suite des fonctions

$$
R_{n}(z)=-\sum_{\nu=1}^{n} \frac{A_{n v}}{\alpha_{n \nu}}-z \sum_{\nu=1}^{n} \frac{A_{n \nu}}{\alpha_{n \nu}{ }^{2}}+\sum_{\nu=1}^{n} A_{n \nu}\left(\frac{1}{z-\alpha_{n \nu}}+\frac{1}{\alpha_{n \nu}}+\frac{z}{\alpha_{n \nu}{ }^{2}}\right)
$$


tend vers la fonction

$$
R(z)=c_{0}+c_{1} z+\sum_{n=1}^{\infty} A_{n}\left(\frac{1}{z-\alpha_{n}}+\frac{1}{\alpha_{n}}+\frac{z}{\alpha_{n}^{2}}\right) .
$$

Done nous ayons

$$
\gamma=\lim _{n \rightarrow \infty} \sum_{\nu=1}^{n} \frac{A_{n v \nu}}{\alpha_{n \nu}{ }^{2}}-\sum_{\nu=1}^{\infty} \frac{A_{\nu}}{\alpha_{\nu}{ }^{2}}, \quad \delta=-\lim _{n \rightarrow \infty} \sum_{\nu=1}^{n} \frac{A_{n \nu}}{\alpha_{n \nu}} .
$$

En appliquant le théorème $I$, on a utilisé le fait que les sommes

$$
\sum_{\nu=1}^{n} \frac{A_{n \nu}}{\left|\alpha_{n \nu}\right|^{2}}
$$

sont uniformément bornées, ce qu' on peut démontrer facilement.

En effet, parce que $z=0$ est un point régulier pour la fonction $R(z)$ il existe un nombre $b>0$, tel que $\left|\alpha_{n,}\right|>b, n>N$. Soit $a<\frac{b}{2}$, alors on a

$$
\frac{A_{n \nu}}{\left|\alpha_{n v}+a\right|^{2}}=\frac{A_{n v}}{\left|\alpha_{n \nu}\right|^{2}} \cdot \frac{\left|\alpha_{n \nu}\right|^{2}}{\left|\alpha_{n y}+a\right|^{2}} \geqq g \frac{A_{n \nu}}{\left|\alpha_{n \nu}\right|^{2}} .
$$

Posons

$$
\begin{gathered}
\frac{1}{\alpha_{n \nu}}=\beta_{n \nu}-i \gamma_{n \nu}, \quad \frac{1}{\alpha_{\nu}}=\beta_{\nu}-i \gamma_{\nu}, \quad \gamma_{n \nu} \geqq 0, \quad \gamma_{\nu} \geqq 0 \\
j(\delta)=\lim _{n \rightarrow \infty} \sum_{\nu=1}^{n} A_{n \nu} \gamma_{n \nu},
\end{gathered}
$$

alors en suivant une marche déjà employée, on démontre que la série $\sum_{\nu=1}^{\infty} A_{\nu} \gamma_{\nu}$ est convergente. Soit $\varepsilon>0$ un nombre arbitrairement petit et le nombre $m$ choisi dè façon tel que l'on ait

$$
\sum_{\nu=m+1}^{\infty} A_{\nu} \gamma_{\nu}<\varepsilon
$$

Soit $\lambda_{m}$ tel que

Pour $m$ fixe on a

$$
\lim _{n \rightarrow \infty} \sum_{\nu=1}^{\lambda_{n}} A_{n \nu} \gamma_{n v}=\sum_{\nu=1}^{m} A_{\nu} \gamma_{\nu} .
$$

$$
j(\delta)=\lim _{n \rightarrow \infty} \sum_{\nu=1}^{\lambda_{n n}} A_{n \nu} \gamma_{n \nu}+\lim _{n \rightarrow \infty} \sum_{\nu=\lambda_{m}+1}^{n} A_{n \nu} \gamma_{n \nu}=\sum_{\nu=1}^{m} A_{\nu} \gamma_{\nu}+g
$$

où

$$
g=\lim \underset{\nu=\lambda_{m}+1}{\sum_{\Sigma}^{n}} A_{n y} \gamma_{n \nu} \geqq 0
$$


done

$$
j(\delta) \geqq \sum_{x=1}^{\infty} A_{\gamma} \gamma_{\gamma}-\varepsilon
$$

pour chaque $\varepsilon>0, c^{\prime}$ est-à-dire

ce qu'il fallait démontrer.

$$
j(\delta) \geqq-\sum_{y=1}^{\infty} j\left(\frac{A_{y}}{\alpha_{y}}\right),
$$

On peut facilement démontrer que $\gamma$ est réel et non négatif. Nous arons

$$
\gamma=\lim _{n \rightarrow \infty} \sum_{\nu=1}^{n} A_{n \nu}\left(\beta_{n \nu}{ }^{2}-\gamma_{n \nu}{ }^{2}-2 i \beta_{n \nu} \gamma_{n \nu}\right)-\sum_{\nu=1}^{\infty} A_{y}\left(\beta_{\nu}{ }^{2}-\gamma_{y}{ }^{2}-2 i \beta_{y} \gamma_{\nu}\right)
$$

d'où

$$
-\frac{1}{2} j(\gamma)=\lim _{n \rightarrow \infty} \sum_{\nu=1}^{n} A_{n \nu} \beta_{n \nu} \gamma_{n \nu}-\sum_{\nu=1}^{\infty} A_{\nu} \beta_{\nu} \gamma_{\nu}
$$

Nous avons démontré que la somme $\sum_{\nu=1}^{n} A_{n \nu} \gamma_{n \nu}$ est bornée et la série $\sum_{\nu=1}^{\infty} A_{\nu} \gamma_{\nu}$ est convergente. Soit $\varepsilon>0$ un nombre arbitrairement petit et soit $m$ choisi de façon que l'on ait $\left|\beta_{\nu}\right|<\varepsilon$, pour $\nu>m$ ce qui est possible parce que $\alpha_{y} \rightarrow \infty$. Soit $\sum_{y=1}^{\lambda_{m}} A_{n v} \beta_{n v} \gamma_{n \nu}$ la somme qui tend vers $\sum_{\nu=1}^{m} A_{\nu} \beta_{\nu} \gamma_{\nu}$. Pour $n$ assez grand nous aurons aussi $\left|\beta_{n y}\right|<\varepsilon, v>m$. Alors nous avons

où

$$
\begin{aligned}
-\frac{1}{2} j(\gamma) & =\lim _{n \rightarrow \infty} \sum_{\nu=1}^{\lambda_{m}} A_{n \nu} \beta_{n \nu} \gamma_{n \nu}-\sum_{\nu=1}^{m} A_{\nu} \beta_{\nu} \gamma_{\nu}+\lim _{n \rightarrow \infty} g_{n}=\lim _{n \rightarrow \infty} g_{n} \\
\left|g_{n}\right| & =\lim _{n \rightarrow \infty}\left|\sum_{\nu=\lambda_{m}+1}^{n} A_{n \nu} \beta_{n \nu} \gamma_{n \nu}-\sum_{\nu=m+1}^{\infty} A_{\nu} \beta_{\nu} \gamma_{\nu}\right| \leq 2 \varepsilon k,
\end{aligned}
$$

$$
\sum_{\nu=1}^{n} A_{n \nu} \gamma_{n \nu}<k, \quad \sum_{y=1}^{\infty} A_{\nu} \gamma_{\nu}<k
$$

$k$ est un nombre fini. Donc nous avons $j(\gamma)=0$. Par conséquent on a

$$
\gamma=\lim _{n \rightarrow \infty} \sum_{\nu=1}^{n} A_{n \nu}\left(\beta_{n \nu}{ }^{2}-\gamma_{n \nu}{ }^{2}\right)-\sum_{\nu=1}^{\infty} A_{y}\left(\beta_{\nu}{ }^{2}-\gamma_{\nu}{ }^{2}\right)
$$

En suivant une marche déjà employée, on démontre que

$$
\lim _{n \rightarrow \infty} \sum_{\nu=1}^{n} A_{n \nu} \gamma_{n \nu}{ }^{2}=\sum_{\nu=1}^{\infty} A_{\nu} \gamma_{\nu}{ }^{2}
$$

en vertu du fait que la somme $\sum_{y=1}^{n} A_{n \nu} \gamma_{n \nu}$ est uniformément bornée et que 
$\gamma_{\nu} \rightarrow \infty$. La série

est convergente, puisque

$$
\sum_{y=1}^{\infty} A_{y} \beta_{y}^{2}
$$

$$
\sum_{\nu=1}^{\infty} A_{\nu} \beta_{\nu}{ }^{2} \leqq \sum_{\nu=1}^{\infty} \frac{A_{\nu}}{\left|\alpha_{\nu}\right|^{2}}
$$

Soit $\varepsilon>0$ un nombre arbitrairement petit et choisi de façon que l'on ait

$$
\sum_{\nu=m-1}^{\infty} A_{\nu} \beta_{\nu}^{2}<\varepsilon
$$

Nous avons démontré qu'il existe des nombres $\lambda_{m}$ tels que

Nous avons alors

$$
\lim _{n \rightarrow \infty} \sum_{\nu=1}^{\lambda_{m}} A_{n v} \beta_{n \nu}{ }^{2}=\sum_{\nu=1}^{m} A_{\nu} \beta_{\nu}{ }^{2} .
$$

$$
\gamma=\lim _{n \rightarrow \infty} \sum_{\nu=1}^{n} A_{n \nu} \beta_{n \nu}{ }^{2}-\sum_{y=1}^{\infty} A_{y} \beta_{\gamma}{ }^{2}=\lim _{n \rightarrow \infty} \sum_{\nu=\lambda_{n}+1}^{n} A_{n v} \beta_{n \nu}{ }^{2}-\sum_{\nu=m+1}^{\infty} A_{\nu} \beta_{y}{ }^{2} \geq-\varepsilon,
$$

pour chaque nombre $\varepsilon>0$, c'est-à-dire $\gamma \geqq 0$ et la première partie $d u$ théorème est démontrée.

Maintenant nous démontrerons la partie inverse du théorème. Supposons done que la fonction $R(z)$ ait la forme (12), où $\gamma$ est un nombre réel non négatif, $j\left(\alpha_{y}\right) \geqq 0$, la série $\sum_{n=1}^{\infty} j\left(\frac{A_{\nu}}{\alpha_{\nu}}\right)$ est convergente, et

$$
j\left(\delta+\sum_{n=1}^{\infty} \frac{A_{n}}{\alpha_{n}}\right) \geqq 0, \quad A_{n}>0
$$

la série $\sum_{n=1}^{\infty} \frac{A_{n}}{\left|\alpha_{n}\right|^{2}}$ étant convergente. Désignons par

$$
B_{p}=\delta+\sum_{y=1}^{p} \frac{A_{\nu}}{\alpha_{\nu}}
$$

Soit $m_{p}$ un nombre qui tend vers l'infini avec $p$, tel que l'on ait

$$
\lim _{p \rightarrow \infty}\left(\frac{m_{p}}{z+\frac{m_{p}}{B_{p}}}+\sum_{\nu=1}^{p} \frac{A_{\nu}}{z-\alpha_{\nu}}\right)=\delta+\sum_{n=1}^{\infty} A_{n}\left(\frac{1}{z-\alpha_{n}}+\frac{1}{\alpha_{n}}\right)
$$

On pent facilement voir que ceei est toujours possible. En effet (13) est equivalente à

$$
\lim _{p \rightarrow \infty}\left(\frac{m_{p}}{z+\frac{m_{p}}{B_{p}}}-B_{p}\right)=0
$$


puisque

$$
\lim _{p \rightarrow \infty} \sum_{n=p+1}^{\infty} A_{n}\left(\frac{1}{z-\alpha_{n}}+\frac{1}{\alpha_{n}}\right)=0
$$

Or (14) est équivalente à

$$
\lim _{p \rightarrow \infty} \frac{B_{p} z}{z+\frac{m_{p}}{B_{p}}}=0
$$

ce qui est vrai par exemple si $m_{p} \rightarrow \infty, m_{p}>\left|B_{p}\right|^{3}$. Posons alors, si $\gamma \neq 0$,

et si $\gamma=0$

$$
R_{p}(z)=\frac{p}{z-\sqrt{\frac{2 p}{\gamma}}}+\frac{p}{z+\sqrt{\frac{2 p}{\gamma}}}+\frac{m_{p}}{z+\frac{m_{p}}{B_{p}}}+\sum_{n=1}^{p} \frac{A_{n}}{z-\alpha_{n}},
$$

$$
R_{p}(z)=\frac{m_{p}}{z+\frac{m_{p}}{B_{p}}}+\sum_{n=1}^{p} \frac{A_{n}}{z-\alpha_{n}}
$$

La suite $R_{p}(z)$ tend uniformément vers la fonction $R(z)$. Parce que

$$
j\left(B_{p}\right)=j(\hat{)})+\sum_{n=1}^{p} j\left(\frac{A_{n}}{\alpha_{n}}\right)=j(\delta)+\sum_{n=1}^{\infty} j\left(\frac{A_{n}}{\alpha_{n}}\right)-\sum_{n=p+1}^{\infty} j\left(\frac{A_{n}}{\alpha_{n}}\right) \geqq 0,
$$

tous les pôles de $R_{p}(z)$

$$
\pm \sqrt{\frac{2 p}{\gamma}}, \quad-\frac{m_{p}}{B_{p}}, \quad \alpha_{n}(n=1,2, \ldots, p)
$$

ont leur partie imaginaire non négative et le théorème est démontré.

En suivant la mème marche de démonstration, on peut obtenir le théorème suivant:

IV'. Soit la suite de fractions

$$
R_{u}(z)=-g_{n}+\sum_{\nu=1}^{n} \frac{A_{n \nu}}{z-\alpha_{n \nu}}, \quad A_{x \nu \nu}>0, \quad j\left(-g_{n}\right) \geqq 0, \quad j\left(\alpha_{n \nu}\right) \geq 0,
$$

qui tend vers la fonction méromorphe $\mathrm{R}(\mathrm{z})$. Alors $\mathrm{R}(\mathrm{z})$ a la forme (12). Celte condition est aussi suffisante.

On peut aussi démontrer des théorèmes analogues pour la limite de fractions de la forme

$$
R_{n}(z)=\sum_{\nu=1}^{n} \frac{A_{n v}}{z-\alpha_{n v}}
$$

$\alpha \leqq \arg A_{n y} \leqq \beta, \beta-\alpha<\pi$, en faisant des hypothèses convenables pour les pồles $\alpha_{n y}$. 
Nous démontrerons maintenant, comme application, quelques théorèmes connus. Dans la note citée, M. Montex considère des fonctions méromorphes qui sont des limites uniformes des fractions rationnelles aveo des zéros et des pòles entrelacés sur l'axe réel. Soit

$$
R_{n}(z)=\frac{P_{n}(z)}{Q_{n}(z)}, \quad \lim _{n \rightarrow \infty} R_{n}(z)=R(z)
$$

une telle suite, où $P_{n}(z), Q_{n}(z)$ sont de degré $n$, et supposons par exemple que les zéros suivent les pôles. On aura

$$
R_{n}(z)=A_{n}+\frac{A_{n 1}}{z-\alpha_{n 1}}+\frac{A_{n 2}}{z-\alpha_{n, 2}}+\ldots+\frac{A_{n n}}{z-\alpha_{n n}}, \quad \alpha_{n \nu}<\alpha_{n, y+1},
$$

En posant

$$
z=-\infty, \alpha_{n_{1}}-\varepsilon, \alpha_{n_{1}}+\varepsilon, \alpha_{n_{2}}-\varepsilon, \alpha_{n_{2}}+\varepsilon, \ldots, \alpha_{n_{n}}+\varepsilon, \infty,
$$

où $\varepsilon>0$ est un nombre assez petit, on voit facilement que les nombres

$$
-A_{11}, A_{u_{1}}, A_{\mu_{2}}, \ldots, A_{\mu n}
$$

doivent avoir le mème signe. Si $\alpha_{n \nu}>0$ en appliquant le théorème III' où $A$ coincide avec l'axe réel positif, on obtient que $R(z)$ a la forme

$$
R(z)=-A+\sum_{i=1}^{\infty} \frac{A_{i}}{z-\alpha_{i}}
$$

où $\alpha_{i}>0, A$ et $A_{i}$ sont de même signe et la série $\sum_{i=1}^{\infty} \frac{A_{i}}{\alpha_{i}}$ est convergente, ce qui est le premier théorème de M. Montex. Si nous supposons maintenant que les pôles $\alpha_{u y}$ sont seulement réels, en applicant le théorème $I V^{\prime}$ pour les deux cas $j\left(\alpha_{n v}\right) \geqq 0, j\left(\alpha_{n y}\right) \leqq 0$ (puisque $j\left(\alpha_{n v}\right)=0$ ) on obtient que $R(\approx)$ a la forme suivante

$$
P(z)=A-B z+\sum_{n=1}^{\infty} A_{n}\left(\frac{1}{z-\alpha_{n}}+\frac{1}{\alpha_{n}}\right)
$$

$A_{n}, B$ sont de mème signe, $\alpha_{n}$ réés, la série $\underset{n=1}{\infty} \frac{A_{n}}{\alpha_{n}{ }^{2}}$ étant convergente, ce qui est l'autre théorème de M. Montel.

Soit

$$
P_{n,}(z)=\left(1-\frac{z}{\alpha_{n_{1}}}\right)\left(1-\frac{z}{\alpha_{n z}}\right) \ldots\left(1-\frac{z}{\alpha_{n n}}\right)
$$

un suite de polynomes qui tend uniformément vers une fonction entière $F(z)$. Alors la suite des fractions

$$
R_{n}(z)=\frac{P_{n}^{\prime}(z)}{P_{n}(z)}=\sum_{\nu=1}^{n} \frac{1}{z-\alpha_{n \nu}}
$$


tend uniformément (dans notre sens) vers la fonction méromorphe

$$
R(z)=\frac{F^{\prime}(z)}{F(z)}
$$

En applicant alor's le théorème III' $^{\prime}$ et $I^{\prime}$ on obtient les théorèmes de MM. Pótya, Schur $\left({ }^{6}\right)$ pour les fonctions entières qui sont limites de polynomes aveo des zéros réels.

Supposons que les zéros des polynomes $P_{n}(z)$ se trouvent dans un secteur $A$ de sommet $z=0$ et d'ouverture $₹ \pi$. D'après le théorème III, on obtient que la fonction entière $F(z)$ a la forme suivante

$$
F(z)=e^{-\delta z} \prod_{n=1}^{\infty}\left(1-\frac{z}{\alpha_{n}}\right)
$$

où $\alpha_{n}$ sont des points de $A, \delta$ est un point de $\bar{A}, \sum_{n=1}^{\infty} \frac{1}{\left|\alpha_{n}\right|}$ est convergente, ce qui est un théorème de M. PóLYa (7).

Supposons maintenant que les zéros des $P_{n}(z)$ se trouvent an dessus de l'axe réel. Alors d'après le théorème III la fonction $F(z)$ aura la forme suivante

$$
F(z)=e^{-\gamma z^{2}+\delta z} \prod_{n=1}^{\infty}\left(1-\frac{z}{\alpha_{n}}\right) e^{\frac{z}{\alpha_{n}}}
$$

où $j\left(\alpha_{n}\right) \geqq 0, \gamma$ réel et non négatif, la série $-\sum_{n=1}^{\infty} j\left(\frac{1}{\alpha_{n}}\right)$ est convergente et sa somme est $\leqq j(\delta), \sum_{n=1}^{\infty} \frac{1}{\left|\alpha_{n}\right|^{2}}$ est aussi convergente, ce qui est un autre théorème de M. Pólya $\left({ }^{8}\right)$, enoncé par lui sans démonstration.

(6) G. Pócya und I. Schun, Ueber zwei Arten von Faktorenfolgen in der Theorie der algebraischen Gleichungen, "Journal für die reine and angew. Math. », 144 (1914), pp. 89-113. E. Lindwart und $G$. PóLYa, Ueber einen Zusammenhang zuischen der Konvergenz von Polynomenfolgen und der Verteilung ihrer Wuraeln, "Rendieonti del Circolo Mat. di Palermo », 37 (1914), pp. 1-8.

(7) G. PбLYa, Ueber Annäherung durch Polynomen, deren sömtliche Wurzeln in einem Winkelraum fallen, "Gött. Nachrichten", (1913), pp. 326-330.

(8) G. Póxya, Su* les opérations fonetionnelles linéaires échangeables avec la dérivation et sur les zéros des polynomes, "Comptes Rendus", 183 (1926), p. 413. 